\title{
SENTINEL LYMPH NODE BIOPSY IN VULVAR CANCER - LITERATURE MINIREVIEW
}

\author{
Alin BODOG ${ }^{1}$, Nicolae BACALBASA ${ }^{2,3,4} \bowtie$, Irina BALESCU5 , Ioan SUCIU ${ }^{6}$, Mihaela VILCU ${ }^{3,4}$, \\ Iulian BREZEAN ${ }^{3,4}$ \\ ${ }^{1}$ Oradea Medical University, Romania \\ ${ }^{2}$ Center of Excellence in Translational Medicine, Fundeni Clinical Institute, Bucharest, Romania \\ 3 "Carol Davila“ University of Medicine and Pharmacy, Bucharest, Romania \\ ${ }^{4}$ „Ion Cantacuzino“ Clinical Hospital, Bucharest, Romania \\ ${ }^{5}$ Ponderas Academic Hospital, Bucharest, Romania \\ ${ }^{6}$ Emergency University Hospital, Bucharest, Romania
}

\begin{abstract}
Vulvar cancer is a rare gynecologic malignancy usually amenable to surgery, especially in cases diagnosed in early stages of the disease. Although initially it had been considered that extended radical surgery should be routinely performed in such cases, the high rates of perioperative complications led to the modification of the therapeutic protocol, more limited surgical procedures being proposed. The extent of the surgical procedure was modified in terms of both vulvar resections and extent of lymph node dissection, sentinel lymph node dissection being widely recommended in the last decade. This is a literature review of the most consistent studies conducted on the theme of sentinel lymph node biopsy for early stage vulvar cancer patients.
\end{abstract}

Keywords: vulvar cancer, sentinel lymph node, biopsy.

\section{RÉsumÉ}

Biopsie du ganglion sentinelle dans le cancer vulvaire - minirevue de la littérature

Le cancer vulvaire est une tumeur maligne gynécologique rare qui peut généralement faire l'objet d'une intervention chirurgicale, en particulier dans les cas diagnostiqués dans un stade précoce de la maladie. Bien que l'on ait initialement envisagé de procéder systématiquement à une chirurgie radicale étendue dans de tels cas, les taux élevés de complications per-opératoires ont entraîné une modification du protocole thérapeutique, des procédés chirurgicaux plus limités étant proposés. L'étendue de la procédure chirurgicale a été modifiée en termes de résections vulvaires et d'étendue de la dissection des ganglions lymphatiques, la dissection des ganglions sentinelles étant largement recommandée au cours de la dernière décennie. Il s'agit d'une revue de la littérature des études les plus cohérentes conduites sur le thème de la biopsie du ganglion sentinelle chez 
des patients atteints d'un cancer de la vulve dans un stade précoce.

Mots-clés: Cancer de la vulve, ganglion sentinelle, biopsie.

\section{INTRODUCTION}

The principles of sentinel lymph node biopsy were initially investigated in penile carcinoma patients, and, later on, applied on wide populations diagnosed with various malignancies having different locations ${ }^{1}$. The rationale of sentinel lymph node biopsy usage is practically the same irrespective of the type of malignancy in which it is used: due to the fact that the largest amount of tumors spread via the lymphatic system, surgeons tried to identify which is the first lymphatic station in which metastatic cells will be delivered; according to this theory, in early stage malignancies, identification and biopsy of this first lymphatic station should be enough in order to assess the status of the surrounding lymphatic stations $^{2-4}$. Therefore, this first station in which is believed that a tumor will lead to the apparition of lymph node metastases is called sentinel lymph node; in consequence, sentinel lymph node biopsy has been proposed in order to assess the lymphatic status $^{5}$. The method is particularly useful in order to minimize the risk of unnecessary extended lymph node dissection and the risk of subsequent morbidity $\mathrm{y}^{6-9}$. When it comes to vulvar cancer, it represents an uncommon gynaecologic malignant tumor with a reported incidence of 4850 new diagnosed cases in the United States of America in 2014 and 1030 vulvar cancer related deaths ${ }^{10}$. This malignancy had been initially treated by local resection followed by inguinal-femoral lymph node dissection; however, the unacceptable rate of early postoperative complications (such as wound dehiscence) or late postoperative complications (such as chronic lower limb lymphedema) lead to the necessity of developing a novel technique in order to decrease the rates of postoperative complications ${ }^{11-19}$. Moreover, a significant number of patients submitted to inguinal-femoral lymph node dissection for vulvar cancer (more than two thirds) present negative lymph nodes; therefore, in all these cases performing sentinel lymph node biopsy seems to decrease the rate of unnecessary extended lymph node dissections ${ }^{20}$. In this way the concept of sentinel lymph node biopsy has become part of the therapeutic strategies of these cases ${ }^{21}$. Moreover, the method of sentinel lymph node biopsy in vulvar cancer patients is particularly feasible and reproductive due to the fact that usually the vulvar region has a predictable anatomy of the lymphatic drainage ${ }^{22}$.

\section{Studies Reporting the SAFETy OF SENTINEL NODE BIOPSY IN VULVAR CANCER PATIENTS}

The first study which demonstrated the effectiveness of sentinel lymph node biopsy in early stage vulvar cancer patients was conducted by Van der Zee and was published by the American Society of Clinical Oncology in $2008^{23}$. The study was a multicentric, prospective one and involved 403 patients with early stage (smaller than $4 \mathrm{~cm}$, squamous cell) vulvar carcinomas submitted to sentinel lymph node biopsy from March 2000 until June 2006 in 15 centers. The postoperative follow-up was provided by visits every two months for the first two years and consisted of interview, clinical examination as well as groin palpation; in the meantime the association of lymphedema and erysipelas was investigated, the presence of lymphedema at two consecutive visits after one year as well as recurrent erysipelas being considered as long-term morbidity causes. Among the 403 cases, unilateral sentinel lymph node biopsy was performed in 183 cases, while in the remaining 220 cases the procedure was bilaterally performed; among these cases, $26.2 \%$ presented positive sentinel lymph nodes (in 163 groins respectively).

Patients presenting positive sentinel lymph nodes were further submitted to complete inguinal-femoral lymph node dissection and developed a significantly higher short-term and long-term morbidity rates when compared to those submitted to sentinel lymph node biopsy; moreover, those who also necessitated association of adjuvant radiation therapy reported a significantly higher number of episodes of erysipelas when compared to those in whom radiation therapy was not delivered. Moreover, patients submitted to sentinel lymph node biopsy reported a median hospital in stay of only 8.4 days when compared to those submitted to complete lymph node dissection (13.7 days, $p<0.0001)$. Among the 139 cases presenting initially negative sentinel lymph nodes, two cases developed groin recurrence within a short interval; however, both cases initially presented multifocal lesions which were separately injected. Later on the authors decided not to include any other case with multifocal lesions. 
After a median follow-up of 35 months, eight cases developed groin recurrence (including the two cases with multifocal disease) after a median time of 12 months. The eight cases were further treated by bilateral complete inguinal-femoral lymph node dissection followed by adjuvant chemo (radiation), local control of the disease being achieved in four of the eight cases. However, six of the eight patients died of disease, while the remaining cases were alive with no sign of recurrence at 50 and 6 months respectively. The explanations for failure consisted of: removing a single lymph node whereas the lymphoscintigraphic examination revealed the presence of two sentinel nodes (in two cases) and missing the presence of micro-metastatic disease (which was detected at re-evaluation of the specimen) - in two other cases. In the remnant four cases, no explanation was provided; however, two cases presented multifocal lesions. At the end of the follow-up period, $5.8 \%$ of cases died due to intercurrent diseases and $3.6 \%$ of cases died due to vulvar cancer progression. In patients with unifocal lesions and negative sentinel lymph node biopsy, the 3 -year overall survival rate was $97 \%$. In conclusion, the study demonstrated the safety and efficacy of sentinel node biopsy in early stage, unifocal vulvar cancer patients; in the meantime, the authors underlined the fact that cases presenting multifocal lesions should not be routinely submitted to this approach $^{23}$.

Four years later, the Gynaecologic Oncology Group Study conducted by Levenbach et al conducted a similar study which was known under the name of GOG17324. The study included 418 patients who had at least one sentinel lymph node identified; in all cases isosulfan blue was injected intradermally in the close proximity of the tumor or in the scar (if the tumor had been previously excised) and five minutes later groin incision was performed. A lymph node was considered as sentinel if a blue channel led to it, even in the eventuality that the lymph node itself wasn't blue. In certain cases, the dual method was used, a radioactive node being considered whether its activity was at least ten times greater when compared to the background radioactivity. Among the 418 cases the incidence of inguinal lymph node metastases was $31.6 \%$ and was strongly correlated with the dimension of the tumor $(p=0.0029)$. As for the characteristics of the sentinel lymph node, among the 418 cases, which presented at least one sentinel lymph node at surgery, the node was both blue and hot in 254 cases, only blue in 100 cases and only hot in 64 cases; in the meantime, the mean number of sentinel lymph nodes per groin was 1.5 . When it comes to the site of injection (in the tumor or at the level of the scar if the primary tumor had been previously resected), it did not influence the rate of sentinel lymph node detection. The authors also reported a sensitivity rate of $91.7 \%$ and a false negative predictive value of $3.7 \%$; moreover, in patients with tumors smaller than $4 \mathrm{~cm}$, the false negative predictive value was $2 \%{ }^{24}$.

Recently, a multicenter observational study GROINSS-V II which aims to determine whether radiotherapy is a safe alternative for lymphadenectomy in early stage vulvar cancer patients with a positive sentinel node, was ended. The study registered 1715 patients between December 2005 and October 2016; however the results are waited to be published at the end of the year $2018^{25}$.

\section{Sentinel node biopsy versus complete Lymph- ADENECTOMY IN EARLY VULVAR CANCER PATIENTS}

When it comes to the surgical therapeutic strategies for vulvar cancer, they evolved from en bloc resection of the vulvar area and inguinal-femoral lymph node dissection to resection and lymph node dissection through separate incisions. Once the protocol was changed, the rate of postoperative complications such as wound dehiscence decreased; however the rate of lower limb lymphedema remained high ${ }^{23}$. Although initially lymphedema did not seem to be such an invalidating problem, later on it proved to have a significant impact on the patients' capability to work, to perform daily activities and to have a normal social life; moreover, in the study conducted by Ryan et al the authors demonstrated that more than one quart of women with chronic lymphedema, considered that the costs of compression garments was a real burden, while one half of cases reported the necessity of modification of their daily program due to the presence of lymphedema ${ }^{26}$.

In order to demonstrate that sentinel lymph node biopsy is more effective than extended lymph node dissection in early stage vulvar cancer patients, McCann et al conducted a study in which they compared the two methods in terms of effectiveness as well as in terms of costs. The study included two categories of patients: those who were submitted to radical vulvectomy and complete inguinal lymph node dissection, and those submitted to radical vulvectomy and sentinel node biopsy. Criteria of inclusion in the study were represented by vulvar lesions smaller than $6 \mathrm{~cm}$ and the absence of clinical suspicion of inguinal-femoral adenopathies ${ }^{27}$.

According to the intraoperative aspects, the patients were further classified in five categories (the first three categories originating from the group of patients who were planned for sentinel lymph node biopsy, while the latter two groups originated from the category of per-primam complete lymph node 
dissection): 1 - patients in whom sentinel lymph node biopsy was planned but in whom the procedure was aborted due to the presence of grossly positive lymph nodes, 2 - patients with negative sentinel lymph nodes in whom no further radiation therapy was necessary, 3 - patients with positive sentinel lymph nodes in whom completion lymph node dissection followed by adjuvant radiation therapy was performed, 4 - patients submitted to per primam lymph node dissection who proved to have positive lymph nodes and were therefore submitted to adjuvant radiation therapy, and 5 - patients submitted to per primam lymph node dissection with negative lymph nodes and therefore, with no need for adjuvant radiation therapy. The authors reported a probability of 5 -year survival rate of $48 \%$ for the first category, $94 \%$ for the second category, $53 \%$ for the third category, $78 \%$ for the fourth category and $53 \%$ for the fifth category. In the meantime the probability of lymphedema was $50 \%$ for the first category, $2 \%$ for the second category, $10 \%$ for the third category, $40 \%$ for the fourth category and $50 \%$ for the fifth category. When comparing the cost effectiveness and the quality of life between the two main categories, the authors underlined that sentinel lymph node biopsy patients had an overall cost with $\$ 812$ lower than complete lymph node dissection cases as well as a longer quality adjusted life year survival (4.16 versus 4). In conclusion, the method of sentinel lymph node biopsy proved to be more cost-efficient, more safety and more comfortable for the patient when compared to standard inguinal-femoral lymph node dissection ${ }^{27}$.

\section{Conclusions}

Sentinel lymph node biopsy in early stage vulvar cancer patients seems to be an effective and safe method in order to decrease the risk of short-term and long-term postoperative complications. The method decreases the length of hospital in stay, the rate of lymphedema and erysipelas and offers a good quality of life for these patients without impeding the oncological outcomes. However, it should not be omitted the fact that the method has been successfully tested only for unifocal lesions while patients with multifocal lesions developed groin recurrences after a relatively short follow-up interval. Therefore, for the moment the method should be routinely performed only for the first category of patients. When it comes to the therapeutic strategy to be followed in positive sentinel lymph node patients, the results of the on-going trials are expected for the end of the year of 2018.

\section{Acknowledgements:}

This work was supported by the project entitled „Multidisciplinary Consortium for Supporting the Research Skills in Diagnosing, Treating and Identifying Predictive Factors of Malignant Gynecologic Disorders", project number PN-III-P1-1.2-PCCDI2017-0833.

\section{Compliance with Ethics Requirements:}

„The authors declare no conflict of interest regarding this article“

\section{References}

1. Cabanas RM. An approach for the treatment of penile carcinoma. Cancer 1977; 39(2):456-466.

2. Cusimano MC, Walker R, Bernardini, MQ, et al. Implementing a cervical sentinel lymph node biopsy program: quality improvement in gynaecologic oncology. $J$ Obstet Gynaecol Can 2017; 39(8):659-667.

3. Karakousis CP, Velez AF, Spellman JE Jr, Scarozza J. The technique of sentinel node biopsy. Eur J Surg Oncol 1996; 22(3):271-275

4. Keshtgar MR, Chicken DW, Waddington WA, Raven W, Ell PJ. A training simulator for sentinel node biopsy in breast cancer: a new standard. Eur J Surg Oncol 2005; 31(2):134-140.

5. Alex JC, Krag DN. Gamma-probe guided localization of lymph nodes. Surg Oncol 1993; 2(3):137-143.

6. Hader D, Moss K, Geier N. Sentinel lymph node biopsy using lymphoscintigraphy. AORN 1998; J68(4):572-82.

7. Pu LL, Cruse CW. Lymphatic mapping and sentinel lymph node biopsy for nonmelanoma skin cancers. Surg Oncol Clin N Am 1999; 8(3):527-539.

8. Schneebaum S, Stadler J, Cohen M, Yaniv D, Baron J, Skornick Y. Gamma probe-guided sentinel node biopsy--optimal timing for injection. Eur J Surg Oncol 1998; 24(6):515-519.

9. Bleiweiss IJ. Sentinel lymph nodes in breast cancer after 10 years: rethinking basic principles. Lancet Oncol 2016;7(8):686-692.

10. American Cancer Society. Cancer Facts \& Figures, 2014 Atlanta: American Cancer Society; 2014.

11. Dorney KM, Growdon WB, Clemmer J, et al. Patient, treatment and discharge factors associated with hospital readmission within 30 days after surgery for vulvar cancer. Gynecol Oncol 2017;144(1):136-139.

12. Aviki EM, Esselen KM, Barcia SM, et al. Does plastic surgical consultation improve the outcome of patients undergoing radical vulvectomy for squamous cell carcinoma of the vulva? Gynecol Oncol 2015;137(1):60-65.

13. Wills A, Obermair A. A review of complications associated with the surgical treatment of vulvar cancer. Gynecol Oncol 2013;131(2):467-479.

14. Sullivan SA, Van Le L, Liberty AL, Soper JT, Barber EL. Association between hypoalbuminemia and surgical site infection in vulvar cancers. Gynecol Oncol 2016; 142(3):435-439.

15. Leminen A, Forss M, Paavonen J. Wound complications in patients with carcinoma of the vulva Comparison between radical and modified vulvectomies. Eur J Obstet Gynecol Reprod Biol 2013; 93(2):193-197.

16. Soliman AA, Heubner M, Kimmig R, Wimberger P. Morbidity of inguinofemoral lymphadenectomy in vulval cancer. Scientific World Journal 2012; Article ID 341253. 
17. Cirik DA, Karalok A, Ureyen I, et al. Early and late complications after inguinofemoral lymphadenectomy for vulvar cancer. Asian Pac J Cancer Prev 2015; 16(13):5175-5179.

18. Micheletti L, Preti M. Surgery of the vulva in vulvar cancer. Best Pract Res Clin Obstet Gynaecol 2014; 28(7):1074-1087.

19. De Hullu JA, Van der Zee AG. Surgery and radiotherapy in vulvar cancer. Crit Rev Oncol Hematol 2006; 60(1):38-58.

20. Sedlis A, Homesley H, Bundy BN, et al. Positive groin lymph nodes in superficial squamous cell vulvar cancer: a gynecologic oncology group study. Am J Obstet Gynecol 1987; 156(5):1159-1164

21. Rouzier R, Haddad B, Dubernard G, Dubois P, Paniel BJ. Inguinofemoral dissection for carcinoma of the vulva: effect of modifications of extent and technique on morbidity and survival. J Am Coll Surg 2003; 196(3):442-450.

22. Slomovitz BM, Coleman RL, Oonk MH, van der ZA, Levenback C. Update on sentinel lymph node biopsy for early-stage vulvar cancer. Gynecol Oncol 2015; 138(2):472-477.
23. Van der Zee AG, Oonk MH, De Hullu JA, et al. Sentinel node dissection is safe in the treatment of early-stage vulvar cancer. J Clin Oncol 2008; 26(6):884-889.

24. Levenback CF, Ali S, Coleman RL, et al. Lymphatic mapping and sentinel lymph node biopsy in women with squamous cell carcinoma of the vulva: a gynecologic oncology group study. J Clin Oncol 2012; 30(31):3786-3791.

25. https://gciggroupcom/system/files/2016 June van der Zee slides GROINSS-V II website v DGOG.pdf.

26. Ryan M, Stainton MC, Jaconelli C, Watts S, MacKenzie P, Mansberg T. The experience of lower limb lymphedema for women after treatment for gynecologic cancer. Oncol Nurs Forum 2003; 30(3):417-423.

27. McCann GA, Cohn DE, Jewell EL, Havrilesky LJ. Lymphatic mapping and sentinel lymph node dissection compared to complete lymphadenectomy in the management of early-stage vulvar cancer: A cost-utility analysis. Gynecol Oncol 2015; 136(2):300-304. 